\title{
Urgences
}

\section{L'esprit du lieu en architecture : lieu commun ou lieu de génie ?}

\section{Paul Faucher}

Numéro 17-18, octobre 1987

L'esprit des lieux

URI : https://id.erudit.org/iderudit/025426ar

DOI : https://doi.org/10.7202/025426ar

Aller au sommaire du numéro

Éditeur(s)

Urgences

ISSN

0226-9554 (imprimé)

1927-3924 (numérique)

Découvrir la revue

Citer ce document

Faucher, P. (1987). L'esprit du lieu en architecture : lieu commun ou lieu de génie ? Urgences, (17-18), 119-130. https://doi.org/10.7202/025426ar d'utilisation que vous pouvez consulter en ligne.

https://apropos.erudit.org/fr/usagers/politique-dutilisation/ 


\section{Paul Faucher}

\section{L'ESPRIT DU LIEU EN ARCHITECTURE: LIEU COMMUN OU LIEU DE GÉNIE?}

\section{A TITRE DE PRÉAMBULE}

J'avais préparé une introduction que je trouvais plaisante, que suivait une projection commentée de projets récents de l'atelier d'architecture auquel je suis associé: à la fois pour éclairer en théorie et faire valoir dans la pratique ce qui se greffe en architecture sur cette recherche de l'esprit du lieu.

L'introduction aurait duré une demi-heure et la projection, une heure et quart!... Louise Déry, bien qu'intéressée par la générosité de mon problème, m'a cependant donné sans hésitation un NON gentil et ferme. Quelqu'un d'autre, plus vigoureusement sympathique à l'auditoire, et connaissant probablement ma lourdeur d'écriture, a statué plus radicalement: "Coupe massivement». J'ai négocié quelques minutes avec Paul-André Bourque, qui sait cadrer ses objectifs, j'ai taillé avec souffrance dans l'entrée en matière, j'ai sabré dans les photos et supprimé tout commentaire sur les bâtiments présentés en ne gardant qu'un titre-référence pour chaque projet. Et je jette le tout vers l'auditoire comme une démarche suggestive et obligée plutôt qu'explicative et partagée...

Trop peu de temps physique pour trop d'espaces virtuels!

L'esprit du lieu en Architecture: lieu commun ou lieu du Génie? C'est ainsi que j'avais commencé le résumé de la conférence, ou mettons plutôt du propos, que j'envisageais de développer pour aujourd'hui.

Sans compter cependant sur l'esclavage auquel se livrent les architectes praticiens: celui de la fuite en avant devant les échéances quotidiennes de leur petite usine à produire... Dans cette espèce de collectivisation du retard qui règne dans un bureau d'architecte, il est difficile de s'isoler, de penser en situation de repos donc de distanciation, et de touver le temps de respirer longuement en soi-même, donc d'écrire. Le rythme brouillon de ce type d'action est irréconciliable avec la situaton d'arrêt serein que requiert la réflexion contemplative.

L'esprit du lieu? Encore aurait-il fallu au préalable trouver le lieu de l'esprit!... Donc, fébrilement, nerveusement, au gré des idées 
volatiles qui de temps en temps filtraient à travers la couche plus épaisse du quotidien, j'ai pris des notes, j'ai laissé s'accumuler les impressions, les tentations, j'ai procédé par sédimentations et influences, par analogie et par référence...

Il faut dire qu'en tant que praticien, plus habitué à "faire" qu'à "dire», (à ne pas confondre, quand même, avec «agir» et "penser»: les praticiens vont parfois jusqu'à penser, comme il peut arriver à certains théoriciens de se plaire épisodiquement dans l'action...), je traîne une méfiance viscérale pour les plaisirs savants et après le fait de la dialectique qui tend d'après moi à coincer les pensées dans la structure même des mots.

Je préfère donc considérer les "choses» dans une optique plus sophiste, plus vécue, plus ardue aussi peut-être (pour le conférencier comme pour son auditoire), parce que moins clairement cataloguée, moins rationnellement évidente, mais aussi (toujours d'après moi) plus réelle parce que plus sentie et donc, finalement, plus sensible.

Mon intention première était de lire, outre l'Esprit des Lieux de Durrell et le Genius Loci de Christian Norberg-Schulz, le Quatuor d'Alexandrie au grand complet, et de composer ensuite l'impression du lieu ressenti à la lecture, avec le lieu lui-même, et de m'en servir alors comme référence de parcours pour y opposer systématiquement Rimouski à Alexandrie ou, dans l'autre sens géographique, le Vieux-Québec à Brasilia, que sais-je?

L'esprit du lieu? Encore aurait-il fallu au préalable trouver le lieu de l'esprit!... Donc, fébrilement, nerveusement, au gré des idées volatiles qui de temps en temps filtraient à travers la couche plus épaisse du quotidien, j'ai pris des notes, j'ai laissé s'accumuler les impressions, les tentations, j'ai procédé par sédimentations et influences, par analogie et par référence...

Il faut dire qu'en tant que praticien, plus habitué à «faire" qu'à "dire", (à ne pas confondre, quand même, avec "agir» et "penser»: les praticiens vont parfois jusqu'à penser, comme il peut arriver à certains théoriciens de se plaire épisodiquement dans l'action...), je traîne une méfiance viscérale pour les plaisirs savants et après le fait de la dialectique qui tend d’après moi à coincer les pensées dans la structure même des mots.

Je préfère donc considérer les «choses» dans une optique plus sophiste, plus vécue, plus ardue aussi peut-être (pour le conférencier comme pour son auditoire), parce que moins clairement cataloguée, 
moins rationnellement évidente, mais aussi (toujours d'après moi) plus réelle parce que plus sentie et donc, finalement, plus sensible.

Mon intention première était de lire, outre l'Esprit des Lieux de Durrell et le Genius Loci de Christian Norberg-Schulz, le Quatuor d'Alexandrie au grand complet, et de composer ensuite l'impression du lieu ressenti à la lecture, avec le lieu lui-même, et de m'en servir alors comme référence de parcours pour y opposer systématiquement Rimouski à Alexandrie ou, dans l'autre sens géographique, le Vieux-Québec à Brasilia, que sais-je?

Heureusement que pour vous comme pour moi, mon pragmatisme professionnel m'a retenu de tels excès. Et j'ai laissé également de côté toutes les îles grecques, toute l'Antiquité, toutes les périodes historiques ou préhistoriques de l'expérience humaine. Entouré comme je le suis de géographes et d'historiens, de quoi aurais-je eu l'air! Je n'ai pu cenpendant complètement me démarquer des écrivains puisque l'on exigeait pour les fins de cette conférence que j'écrivis, que j'écrivisse ou que j'écrivasse!

Ayant d'autre part peu pratiqué la théorie, donc l'exercice synthétique qui vise l'essence, et étant plus porté vers la pratique, donc vers le geste analytique qui appréhende la substance, j'ai dû, en catastrophe, modifier toute mon approche: j'aurais bien voulu planer à de hautes altitudes mais j'aurais trop ressemblé à Icare (avec les risques bien connus que cela a comporté pour ce pilote-amateur) et les résultats auraient pu être pour moi similaires à ceux qu'il connut, paraît-il, et donc perdre l'esprit!

Je me contenterai finalement du rase-motte, je considérerai le thème donné plus sous l'angle d'une interrogation sur l'outil que d'un constat sur l'objet, plus près de l'image-effet que de la vision-cause.

Attendez-vous donc plus à l'énumération d'un certain nombre de commentaires alignés à la queue leu leu (ou si j'osais me permettre une pareille platitude: à la queue lieu lieu...), suivie, puisque l'on ne peut parler honnêtement que de ce que l'on connaît bien, par la présentation de diapositives de projets réalisés par notre bureau, où j'aurais tenté de vous montrer par le biais d'exemples concrets les mécanismes divers de la quête de l'esprit des lieux en architecture...

Si j'en avais eu le temps!

Mais j'accepte comme opportune l'obligation où la situation me met de libérer rapidement l'architecture des mots, et de la replacer 
dans son mode naturel de découverte, celui du regard. Mais quel survol rapide vous allez devoir faire! L'express des lieux!

Avant de m'engager dans un type de démarche tendancieuse, je veux parler d'une situation dans laquelle une personne seule, plus éclairée que les autres (je parle uniquement de la répartition des éclairages dans cette salle!...), parle à un auditoire efficacement tenu dans la pénombre et le silence. Je tiens à vous citer un extrait de l'introduction que l'écrivain Doris Lessing a rédigée pour son livre The Golden Notebook et qui s'applique merveilleusement comme mise en garde à tous ceux qui se préparent à écouter un conférencier.

Cet extrait concerne la connaissance. Je cite:

Vous ètes en train de vous faire endoctriner. Nous n'avons pas encore
réussi à mettre au point un système d'éducation qui ne soit pas un
système d'endoctrination. Nous nous en excusons mais c'est le mieux
que nous puissions faire. Ce que vous vous faites dire ici représente un
amalgame de préjudices en vogue et les choix particuliers de cette
société particulière. Le moindre coup d'oeil à l'histoire vous prouvera à
quel point ces attitudes sont précaires. Ceux qui vous enseignent sont
ceux qui ont été capables de s'accomoder d'un régime de pensée établi
par leurs prédécesseurs. Il s'agit d'un système qui se perpétue lui-même.
Ceux d'entre vous qui sont plus robustes et individualistes se sentiront
encouragés à partir et à trouver leur propre voie pour s'éduquer et
éduquer leur propre jugement.

Ceux qui restent döivent se souvenir toujours et en toute occasion qu'ils se font mouler et conformer pour correspondre aux besoins étroits et particuliers de cette société spécifique.

Fin de la citation.

Comme le disait Descartes: "Je pense donc je suis", mais peut-être est-ce plus à prendre dans le sens du verbe "suivre" que dans le sens du verbe "être»! Vous êtes prévenus...

Ayant dit cela, et me sentant sans doute plus confortable de vous avoir peut-être un peu déstabilisé (question d'équilibre!), j'en reviens enfin au sujet qui me déstabilise depuis trop longtemps: «L'esprit du lieu en Architecture».

L'Architecture a été de tout temps l'art-mère. Avec l'ambiguité philosophique majeure par rapport aux autres arts de devoir être physiquement utile..., avec l'ambiguilté additionnelle, qui brouille sa compréhension facile, d'être en même temps le plus concret parce que le plus technique, et le plus abstrait parce que le moins immédiatement représentatif de tous les arts. 
Et il ne me reste que 20 minutes pour vous la rendre familière!

Envisageons alors la chose sur un autre plan et acceptons, au sens le plus large, que le rapport entre la «nature» et la "culture» passe nécessairement par la "sculpture» de la première par la deuxième. Tous les arts sont sculpture, structuration, impression, construction. Tous son architecture... Tous son musique, écriture, danse, dessin... Seule la technique varie. Ainsi que le médium d'expression. L'effet de tous ces gestes est de même rapport entre la nature et la culture.

Carlo Scarpa, architecte contemporain italien (je devrais dire architecte vénitien, tant ce lieu a d'esprit depuis qu'il existe...), Carlo Scarpa donnait cette définition de l'architecture: "L'envie de représenter quelque chose découle essentiellement et finalement du besoin de projeter dans le fixe de la mémoire le caractère passager du vécu ou du à vivre, en acceptant comme compromis la permanence et lunicité de certaines apparences, plutôt que la fugacité et la superposition des réalités successives". Fin de la citation.

Voilà qui place l'architecture non seulement dans les trois dimensions de l'espace mais la situe également dans le temps où elle se fait, moment entre l'avant et l'après.

Je reviens à mon résumé. Immanquablement, cependant, même dans ses démonstrations les plus apparemment discutables, la chose construite est symbiose de temps et d'espace et exprime, abjectement, ordinairement ou exemplairement, l'esprit de l'homme qui l'a conçue et de la société qui l'a générée.

Symbiose, donc, d'espace et de temps, la quatrième dimension de l'architecture: le temps d'y penser en le faisant, le temps d'y circuler en l'utilisant, le temps qu'elle a duré du moment qu'on l'a construite au moment où quiconque l'observe ou l'observera.

La cinquième dimension de l'architecture serait le poids du lieu, l'empreinte à la fois de l'homme sur le lieu et du lieu sur l'homme, de la lisibilité et de l'équilibre symbolique des rapports qui s'établissent entre ces deux données essentielles. En fait, l'on pourrait dire que le lieu naturel n'a pas d'esprit: il n'en a pas besoin. Il lui suffit d'être et de modifier, mystifier, impressionner ou séduire, par l'implacable permanence de son état, entre son propre dedans trop opaque et le dehors trop vide qui l'englobe, les humains qui bougent à peine sur sa surface ou tout près d'elle. 
Pourtant c'est à travers la qualité de la pensée de ce petit homme qui l'observe et l'approprie à son imaginaire que le lieu se définit. MAIS encore-faut-il que cet imaginaire existe qui, seul, peut prendre possession du lieu et, respectueusement et audacieusement, peut lui conférer par une sorte d'échange osmotique cette âme dont on parle.

Il existe une multitude de lieux dont la présence écrasante reste finalement trop vaste pour la médiocrité des ambitions et des moyens humains. Je pourrais en citer bon nombre tout près d'ici... et d'ailleurs.

À l'opposé, la marque par laquelle l'homme identifie sa présence peut-elle s'inscrire sans quelque violence?

Où n'existe pas l'équilibre n'existe pas l'esprit: équilibre d'harmonie, ou équilibre de tension, de contraste ou même d'opposition. L'esprit du lieu n'est pas le lieu comme tel mais l'émanatin de l'espace-temps, inscrite et provoquée par la démarche humaine à son endroit, filtrée et amplifiée par les composantes construites et les rapports que celles-ci exercent entre les espaces qu'elles définissent et ceux qui les entourent, le dedans et le dehors de l'intervention. Le lieu construit par l'homme est par nature le réceptacle symbolique irrémédiable de son état comme de ses avoirs, de ses connaissances comme de ses croyances, et le témoin féroce parce que privilégié des conjonctures collectives de son temps.

L'architecture idéale serait donc, au sens profond, une conjonction d'intentions philosophiques et socio-culturelles AVANT d'être au sens large art, technique, finance et gestion... Entre l'infini et l'indéfini, le fini, puis entre le savoir et le faire, le savoir-faire. L'expression magistrale obligatoire d'un moment de pensée profonde et universelle!

Nous n'avons qu'à regarder autour de nous pour vérifier que le meilleur (en petite quantité) côtoie le pire (en énorme dose) et que l'architecture n'est pas différente des autres activités humaines. Son problème majeur réside cependant dans sa durée et dans une "présence" supérieure aux autres formes d'expression créatrice, et qui rend ses déchets plus visibles et pour plus longtemps.

La sixième dimension de l'architecture résiderait alors dans l'épaisseur même du temps, qui distancie l'objet de son réalisateur, le lui ôte, pour ainsi dire, à cause de cette permanence qu'elle possède et que l'humain n'a pas. Et l'interrogation accumulée des générations 
successives qui l'utilisent sans nécessairement en respecter le caractère d'origine d'ailleurs, ajoute progressivement à ce qui reste de cette architecture d'autrefois, par agrégation presque métaphysique ou même par ignorance des causes premières, une dimension magique: en transmutant la spécificité originale objective de ce lieu construit en une essence subjective rattachée plus à l'espace cosmique du temps qu'à l'espace physique de la matière, à la conscience plus qu'à la sienne, et que l'on déclare alors plus ou moins officiellement être l'esprit, l'âme ou le génie du lieu... Comme si le lieu récupérait alors le génie de l'homme.

Mais les époques de changement et d'instabilité, comme l'a défini John Ruskin dans «Les sept lampes de l'architecture», je crois, présentent une fascination particulière pour les ruines. Et cette fascination référentielle pour le passé, qui a toujours marqué les périodes de transition comme celle que nous vivons actuellement en architecture tout du moins, génère, après l'équilibre apparent et immobile des périodes "classiques", le déséquilibre d'autant plus volontaire et complaisant qu'il est forcené des périodes «romantiques». La prochaine fois que vous passerez par Montréal, jetez un coup d'oeil sur ce qui s'y monte... Une énorme tour rose pâle au couronnement de gros diamant ou de tiare feignant presque élégamment de se faire plus petite derrière une minuscule cathédrale. Des Goldorak de trente étages qui exhibent dans leur peau de granit, entre les replis trop gras de leur abondance, l'indécence étrange d'ouvertures de mursrideaux crénelés dont le couronnement ridicule, en forme évocative de clé de voûte voudrait-on nous faire croire, ressemble plus d'après moi à la tirette démesurée d'une gigantesque fermeture éclair. Et cette fermeture éclair ouverte exhibe à la jeune ville (!) effarouchée les monstruosités et les misères propres à l'exhibitionnisme le plus humainement primaire!

Cela passera sans doute comme la picotte! Bien qu'elle laisse des marques... Mais je me demande quand même s'il n'existe pas à notre époque une perception nouvelle étrangement bidimensionnelle de l'espace et de la réalité: la télévision, le film, la photo ont habitué à l'à-plat, à la seule apparence et à la distanciation par rapport à la réalité de trop de faits.

La vitesse et la linéarité des déplacements s'opposent aujourd'hui à la perception de la progression dans l'espace et de la profondeur du champ qui entoure pourtant, dans toutes les autres directions, le déplacement de tout individu. Cette distanciation et cette vitesse limitent la capacité des gens à communiquer entre eux, amenuisent leur perception de l'espace et nient le passage d'un 
temps que l'on veut retenir.

La terre deviendrait-elle plate (dans tous les sens du terme...)?! Entre le modernisme qui s'achève doucement lui-même et les plus visibles et vulgaires outrances placardées de certains post-quelque chose, où placer l'espoir de l'architecture à venir?

Je dirais, humblement, dans la continuité évolutive et sereine de la simple quête de la spécificité de toute réponse à toute suggestion. Au-delà et au-dessus des modes, vers l'état d'irrémédiabilité idéale qui, entre plusieurs solutions envisageables, mène à la meilleure solution possible. La seule qui puisse engendrer le germe de l'esprit dans la conjonction du lieu où construire, et du lieu à construire, au moment où il se pense et se fait. Cette spécificité peut être éminemment variable. Sa forme de synthèse dépend du lieu même, des clients et de leurs besoins, du contenu du programme fonctionnel, des exigences spatiales comme de la signifiance symbolique de l'enveloppe, de l'orientation comme de l'implantation. Et du budget...

Et du hasard, de l'accident de parcours, de la perception et de l'apprivoisement de la dynamique virtuelle du lieu, de la capacité qu'auront les uns et les autres de faire progresser leurs interlocuteurs dans leur sens personnel, certes, mais pour arriver à un sens commun, donc à une dimension qui les dépasse individuellement, plus loin que s'ils avaient été chacun seuls. Et je pense ici, particulièrement, à la connivence à retisser et resserrer entre l'architecteartiste-visionnaire et les autres-artistes-visionnaires... Et au travail patient et long qui, sur 2 à 5 ans, mène un projet d'architecture de son faisceau d'imaginaires à la fin de sa gestation physique. Mais j'avoue ne pas connaître la recette de cette quête: il s'agit d'une expérience continuellement renouvelée pour chaque projet, et à chaque moment de chaque projet. Il s'agit d'un phénomène évolutif qui «tend vers» un objectif mais "n'arrive jamais absolument à» cet objectif.

Pour clore ce long monologue pressé, il ne me reste qu'à vous livrer quelques projets de notre bureau en espérant vous persuader qu'il existe une connivence d'intention, perceptible par la seule image, entre ce que je vous ai dit et ce que nous faisons, et que l'on peut y découvrir cette quête de la spécificité qui, pour moi, reste le principal support de cette présence de l'esprit accordé au lieu.

Pointe du Moulin à l'Ile-Perrot: du passage progressif dans le temps et de l'usage des axes pour organiser l'espace de la visite. 
Hôpital pédiatrique du Centre hospitalier de Fann à Dakar au Sénégal: des besoins de l'enfance, des malades et de l'Afrique, et d'un essai de mimétisme climatologique et formel.

Centre d'accueil pour personnes âgées Armand-Lavergne à Montréal: à propos d'un interface entre le tissu du quartier et la structure de l'institution.

Musée du Fort Chambly: du médium considéré comme étant le message principal qui doit tout dire ce qu'il peut dire.

Restauration de l'Église Saint-Jean-de-la-Croix à Montréal: à la recherche d'un autre espace et d'une certaine lumière intérieure.

Poste Montagnais pour Hydro-Québec, au Nord de Sept-Iles: du transitoire et de la nordicité.

Bibliothèque municipale de Terrebonne: à la recherche du temps perdu, à la lecture du temps qui passe, avec la connivence de Pierre Leblanc, sculpteur et de René Derouin, graveur et peintre.

500 ouest, rue Sherbrooke: à propos d'un interface entre deux gâchis, celui de la rue et celui du bâtiment existant.

Station de métro du Parc: du mouvement généré par le fixe et de l'animation symbolique de l'espace, avec la collaboration de Huguette Desjardins, peintre coloriste et de Claire Sarrasin, sculpteure.

\section{EN GUISE DE POST-SCRIPTUM}

Pour relancer les commentaires de Doris Lessing qui m'ont servi d'introduction, et pour faire preuve moi aussi d'un grain de sédition, j'ai pensé ne pas conclure.

En vous laissant dans le silence regarder un des rares exemples absolus à exister sur cette planète d'un lieu vivant totalement de son esprit et qui n'a demandé pourtant le recours d'aucun spécialiste: ici pas d'architectes, de sculpteurs, de peintres, de coloristes, de lissiers, de graphistes, de littérateurs ou de penseurs, de géographes ou d'historiens. Il s'agit de cinq petites villes groupées, fondées dans les années 1000 autour d'une vallée du Nord du Sahara algérien transformée en oasis par la volonté de ses habitants, refoulés là par la hargne de leurs voisins. Cette région porte le nom de M'Zab et les villes s'appellent Ghardaïa, Beni-Isguen, Melika, El-Ateuf et BouNoura. Elles sont habitées par une secte islamique puritaine très cohérente, trop cohérente pour subsister encore longtemps dans un monde devenu trop rapide pour sa propre fixité et trop superficiel pour la profondeur de ses traditions.

Ceci pour vous laisser dans «l'ambiguïté des choses» (ce qui n'est pas la même chose que l'absurde). Si cet état de fait peut encore effectivement exister, c'est qu'il découle d'une harmonie absolue 
entre un lieu, un temps et la perfection des systèmes économique, social, politique et philosophique de ses occupants, et d'une rigueur intellectuelle qui correspond à la rigueur du lieu.

Consolons-nous cependant en acceptant que la perfection ne soit justement pas de ce monde et que le fait de trop s'en approcher est déjà, par définition, hâter une déchéance prochaine!

Je sais au moins qu'ici nous n'en sommes pas encore là... 

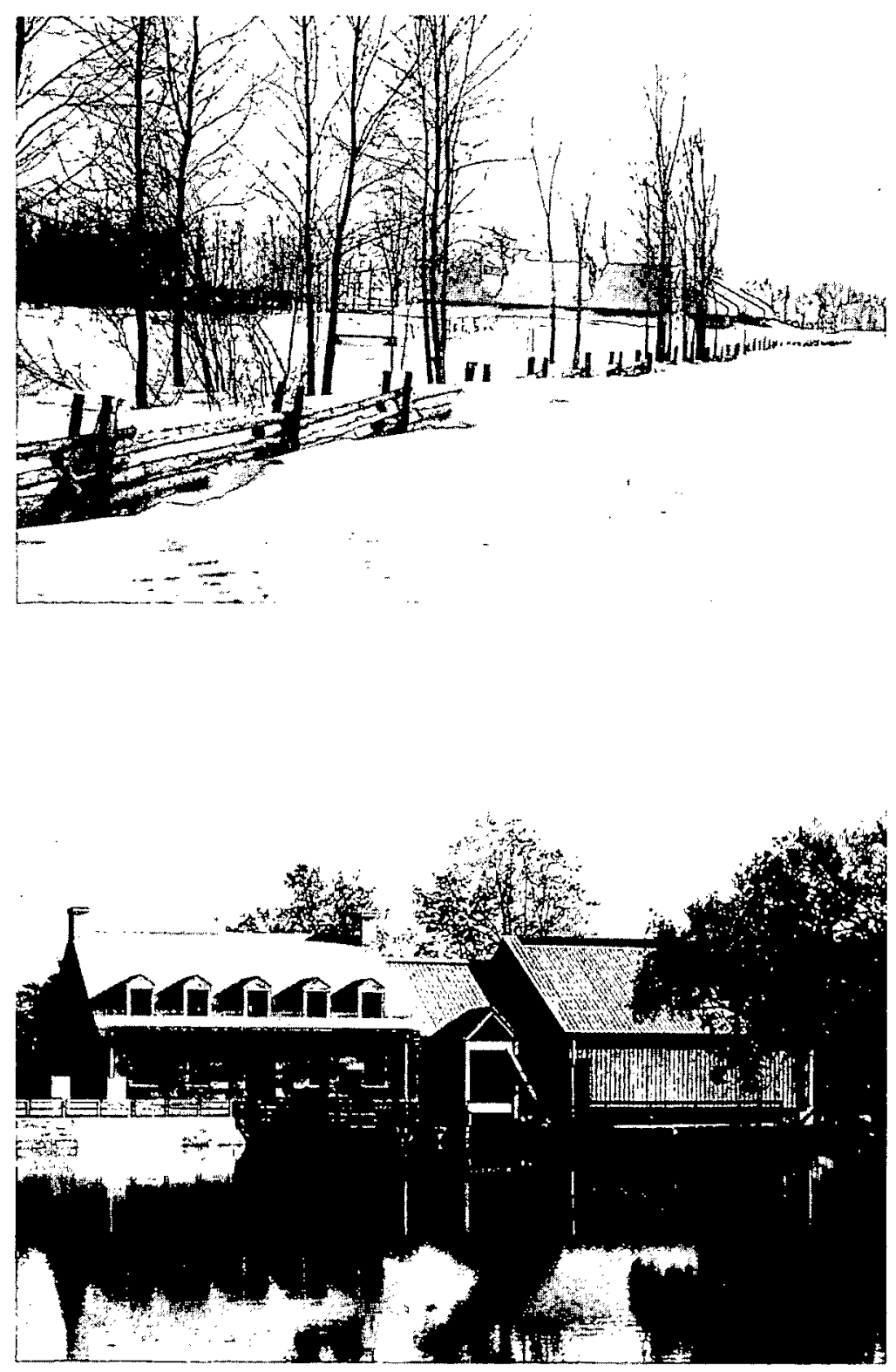

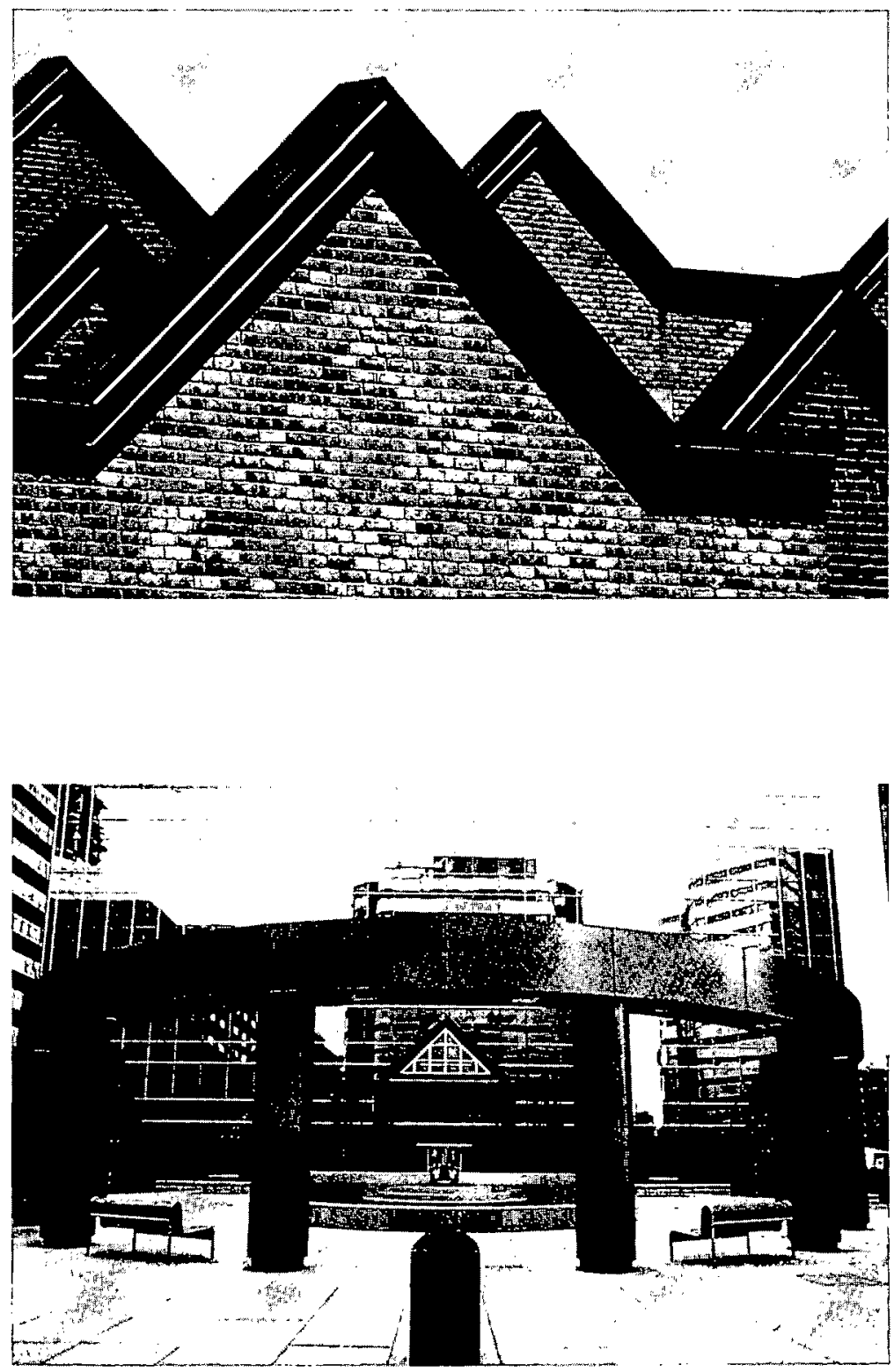